\title{
Simulation Analysis of Contributing Factors to Rider Visibility Issues for Car-Motorcycle Accidents
}

\author{
Danish Farooq ${ }^{1 *}$, Janos Juhasz ${ }^{1}$ \\ ${ }^{1}$ Department of Transport Technology and Economics, Faculty of Transportation and Vehicle Engineering, \\ Budapest University of Technology and Economics, 1111 Budapest, Müegyetem rkp. 3, Hungary \\ * Corresponding author, e-mail: farooq.danish@mail.bme.hu
}

Received: 29 November 2019, Accepted: 07 February 2019, Published online: 16 April 2019

\begin{abstract}
Rider visibility has been considered an important issue in car-motorcycle accidents due to the frequency of unperceptive and negligent driving behavior. Mostly car drivers stated that they didn't see the rider before the collision. This study aims to investigate the contributing factors that reduce rider visibility in point of view of car drivers for car-motorcycle accidents. The study considered the car-motorcycle accidents which occur in the built-up area. Statistical data analysis categorized the accidents into six dominant accident types. Twenty-five accidents were selected by accident types for simulation analysis. The selected accidents were simulated in Virtual Crash software during 5 seconds before the collision. The simulation analysis evaluated that the view obstructions, blind spots and high speed were the contributing factors that reduce rider visibility in car-motorcycle accidents. The simulation plots identified the involvement of contributory factors and variation within the time interval. The comprehensive in-depth analysis also evaluated that no collision avoidance maneuvers were performed by most of the car drivers due to visibility issues before the collision. Safety systems were proposed based on observed factors according to car and motorcycle perspectives for collision avoidance.
\end{abstract}

Keywords

car-motorcycle accidents, contributing factors, rider visibility issues, simulation analysis, safety systems

\section{Introduction}

A visibility limitation has been considered any situation in which the powered two-wheeler (PTW) rider or the other vehicle driver was unable to see the surrounding traffic or was unable to be seen by the surrounding traffic (ACEM, 2004).

Accidents involved specific situations such as "lookedbut-failed-to-see" where the visual perception of related information is disrupted. Several physical and psychophysical constraints on vision could describe the perception failure (Herslund and Jorgensen, 2003). Typically, in car-motorcycle accidents, the car drivers stated that they "looked but failed to see" the oncoming motorcycle. It is possible sometimes that some of these drivers failed to look visual scene in the appropriate areas of the incident (Brown, 2002; Clarke et al., 2007). This phenomenon could be further described such as the road user did not recognize the danger of driving fast in an area with poor visibility or did not decrease their speed in accordance with the right of way (AIB Report, 2009).

Pre-crash events were mostly considered as the main factors in the classification of accident configurations
(Pierini et al., 2004). The TRACE project determined six different situations and at four-legged intersections from a statistical analysis of crashes. The situation where A crosses the road and the path of the opponent vehicle B, which is going straight or turning, is more common and more severe than any other (Molinero Martinez et al., 2007). A typical case was observed which involved a car moving out from a junction into the path of an approaching motorcycle (Clarke et al., 2004).

This paper analyzed mainly contributing factors to rider visibility issues in car-motorcycle accidents. The car-motorcycle accidents were categorized into six dominant accident types by statistical data analysis. Twentyfive accidents were selected for simulation in Virtual Crash software for 5 seconds before the collision. The police report was used as the main data source for selected accidents and simulation models plotted accordingly. Analysis results showed that the view obstructions and blind spots were the main contributing factors to rider visibility issues in car-motorcycle accidents. The impact 
speeds of drivers and riders as well as collision avoidance maneuvers performed by both participants before collision were also examined in the study. Safety systems were proposed based on simulation analysis results to solve rider visibility issues in car-motorcycle accidents.

\section{Research methodology}

\subsection{Statistical data analysis and data sampling}

Real world accident data has been considered important to understand accident characteristics and to identify countermeasures. Car-motorcycle accident data was collected from the Hungarian central statistical office from 2011 to 2014. There were total 2,673 car-motorcycle accidents were observed which involved one car and one motorcycle. The accidents which happened in the built-up area were considered for the study. Statistical data analysis categorized the accidents into six dominant types based on percentage occurrence (rate) for each accident type as shown in Fig. 1. These accident types were described as: (a) car and motorcycle both traveling straight and same direction; (b) car and motorcycle both traveling straight and opposite direction; (c) car and motorcycle both traveling same direction and one turning; (d) car and motorcycle both travelling opposite direction and one turning; (e) car and motorcycle both coming from adjacent direction and without turning; (f) car and motorcycle both coming from adjacent direction and turning. Results showed that the most frequent accident type was vehicles coming from the same direction and one vehicle turning about $23 \%$. The least frequent accident type was vehicles coming from the opposite direction and straight ahead about $4 \%$.

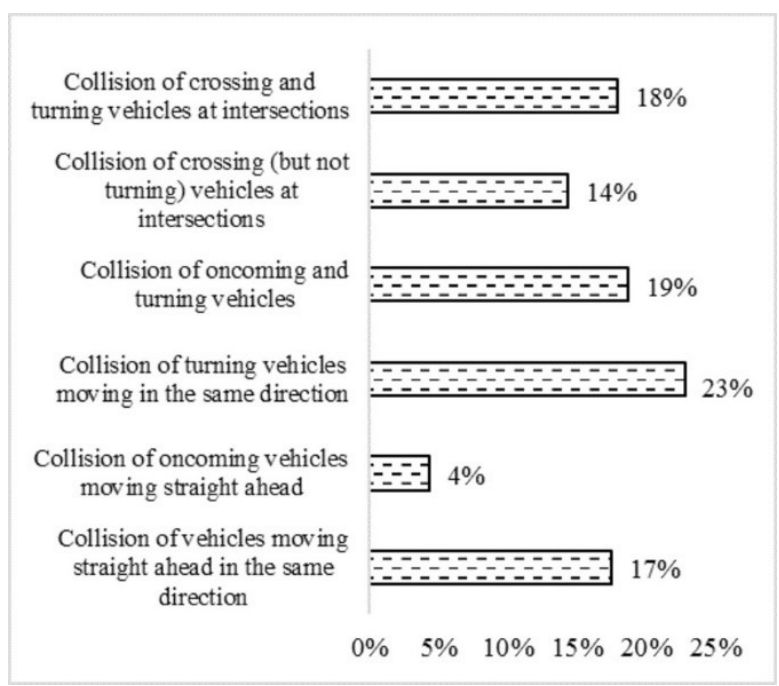

Fig. 1 Percentage of car-motorcycle accidents by accident types in built-up area

\subsection{Virtual crash simulation}

Twenty-five accidents were selected by accident types for simulation analysis. The police report was used as a data source for selected accidents and simulation models plotted according to scale (M 1:200). The simulation model used in the study was shown as an example in Fig. 2. Policereported accident data contain a good deal of valuable information, such as vehicle types, road types, and locations involved (Jacobs et al., 2000). The accident characteristics considered in simulation models include participant's position, sizes, brake, relative heading angle, speed, view obstructions and traffic volume. In order to identify the contributing factors in car-motorcycle accidents, the investigation involved a full reconstruction of the accidents on Virtual Crash software 2.2. The reconstruction of a road vehicle accident is a multidimensional engineering task designed to reconstruct the cause of an event and its course. It can also be understood as a reverse engineering operation (Wach, 2016). Car-motorcycle accidents were simulated based on simulation models for 5 seconds before the collision. This time before the collision has been considered important because drivers/riders must perceive and react according to aware/unexpected situations.

\subsection{Collision configuration and blind spots measurements}

In order to describe the collision configuration and the orientation of the car and motorcycle at the time of collision, the relative heading angles between the car and the motorcycle were measured. The relative heading angle represents the angle between the car and the motorcycle at the time of contact, expressed as a positive angle, clockwise from the vertical where zero degrees corresponds to both vehicles

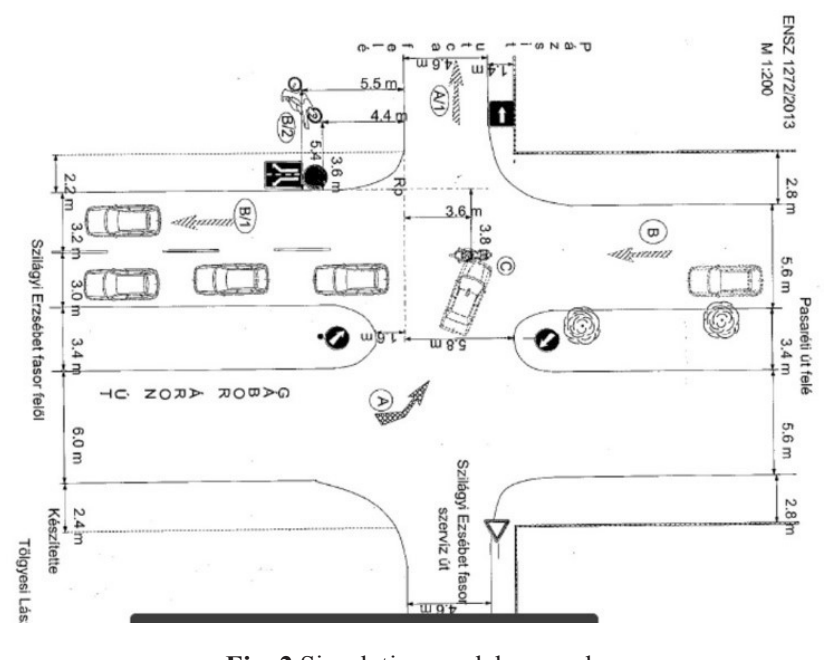

Fig. 2 Simulation model example 
being pointed in the same direction (ACEM, 2004). A blind spot is an area in the range of vision that car driver cannot see properly but which he really should be able to see (Collins Publishers, 2018). These blind spots of a car were measured in a field test using one car and one motorcycle. Fig. 3 showed the observed blind spots region in terms of relative heading angles at moment of collision for specified interval of time. The results showed that about $36 \%$ riders were in blind spots area at moment of collision.

\section{Results and discussions}

From the analysis of simulation results, the observed contributing factors related to rider visibility issues were view obstructions (stationary and mobile), blind spots, impact speed and collision avoidance maneuvers (braking, swerving). All this information increased the accuracy and reliability for in-depth analysis of accidents.

\subsection{View obstruction and blind spots contribution to rider visibility issues}

View obstructions can be defined as the sight of view not visible due to stationary or mobile view obstructions. To observe view obstruction from the driver point of view the camera emulator tool was used on the left, right and top mirror positions in cars in Virtual crash software. Results showed that the stationary view obstructions (buildings, vegetation and parked vehicles) were identified as contributing factors for $28 \%$ accidents and the mobile view obstructions (cars and large buses) were identified as contributing factors for $24 \%$ accidents.

Blind spots in car-motorcycle accidents represented an area in which car driver declared that they failed to see the oncoming rider. Blind spots were measured by considering the range of relative heading angle values (Fig. 3).

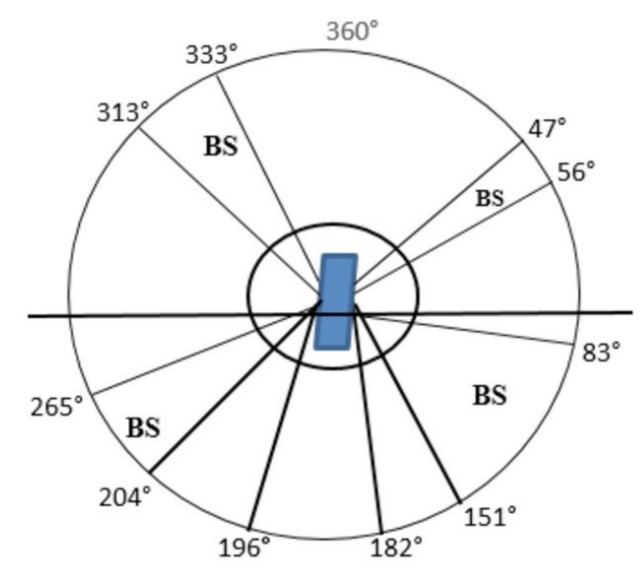

Fig. 3 Blind spots area of a car
The exact position of blind spots for car-motorcycle accidents was described by considering relative heading angle values with respect to time. Simulations were run for each accident on virtual crash software and relative heading angles were measured for each time interval. The selected time interval ( 5 seconds) was considered important because the car drivers and motorcycle riders have to perceive and react before the collision. The reaction time of car drivers was studied, and results determined that it was around $0.70-0.75 \mathrm{~s}$ when fully aware and $1.25-1.5 \mathrm{~s}$ when unexpected situations (Green, 2000). The reaction time of motorcycles under fully aware or unexpected conditions was measured about 0.7-0.9 s (Tang, 2003).

The simulation data plots were developed between relative heading angle and time for all six types of accidents as shown in Fig. 4 (a)-(f). These simulation plots identified the involvement and variations of contributory factors (view obstructions and blind spots) within the specified time period. In these plots, the relative heading angle (degree) was taken along $\mathrm{x}$-axis and time (seconds) was taken along the y-axis. Different marks in the plots provided different type of information such as circular marks represented the visible region; cross marks represented the blind spots region and rectangular marks represented the view obstructions (stationary and mobile) region.

The relation between relative heading angle and time for the accident type in which vehicles coming from the same direction and straight ahead was shown in Fig. 4 (a). The results showed that in most of the cases there were circular marks which identified the visible region in point of view of car drivers and some cases there were cross marks which detected blind spots as contributing factor for the specified time period. Furthermore, the relation between relative heading angle and time for the accident type in which vehicles coming from the opposite direction and straight ahead was shown in Fig. 4 (b). The results showed that for the only case most of the points were rectangular which detected view obstruction as contributing factor and some points were observed circular which identified the visible region for car drivers just before the moment of collision.

The relation between relative heading angle and time for the accident type in which vehicle coming from the same direction and turning was shown in Fig. 4 (c). The results evaluated that in most of the cases there were circular marks which identified the visible region except one case in which most of the points were rectangular which identified the view obstructions as contributing factor to rider visibility issues. In some of the cases, there were 
cross marks which detected blind spots as contributing factor for specified interval of time. The the relation between relative heading angle and time for the accident type in which vehicles coming from the opposite direction and turning was shown in Fig. 4 (d). The simulation results showed that in most of the cases there were circular marks which identified the visible region except two cases in which most of the points were rectangular which detected the view obstructions as contributing factor to rider visibility issues. In one case there were some cross marks which detected blind spots as contributing factor to rider visibility issues for a very short interval of time.

The relation between relative heading angle and time for the accident type in which vehicles coming from an adjacent direction and without turning was shown in Fig. 4 (e). The results showed that in most of the cases there were rectangular marks which detected the view obstructions as contributing factors to rider visibility issues for the long-time interval. In some of the cases there were some cross marks which detected the blind spots as contributing factor to rider visibility issues and there were some circular marks which identified the visible region for a short interval of time. The relation between relative heading angle and time for the accident type in which vehicles coming from an adjacent direction and turning was shown in Fig. 4 (f). The results evaluated that in most of the cases there were rectangular marks which detected the view obstructions as contributing factor to rider visibility issues for a long-time interval. There were some circular points which identified the visible region for a short interval of time. In some case there were some cross marks which detected blind spots as contributing factor to rider visibility issues for a very short interval of time.

\subsection{High speed contribution to rider visibility issues}

Motorcycles' poor conspicuity may be intensified with higher speed, which may decrease their detectability from a turning motorist's perspective (Brenac et al., 2006; Kim and Boski, 2001). Previous studies revealed that the risk of injury increases with impact speed. However, exact impact speeds are not mostly available in most crash reports (Watanabe et al., 2012; Seyer et al., 2000).

The observed speed of car and motorcycle for 5 second before collision and at moment of collision were shown in Table 1. The rider speed was observed as contributing factor from simulation analysis results. The speed of motorcycles was observed higher as compared to cars in most of the cases which affect its visibility. Statistics about the speed distributions by motorcycles and cars found that average motorcycle
Table 1 Car and motorcycle speed information

\begin{tabular}{|c|c|c|c|c|}
\hline \multirow[t]{2}{*}{ No. } & \multicolumn{2}{|c|}{$\begin{array}{c}\text { Speed }(\mathrm{Km} / \mathrm{h}) \text { before } \\
\text { collision }\end{array}$} & \multicolumn{2}{|c|}{$\begin{array}{c}\text { Speed }(\mathrm{Km} / \mathrm{h}) \text { at moment } \\
\text { of collision }\end{array}$} \\
\hline & Motorcycle & Car & Motorcycle & Car \\
\hline 1 & 59 & 47 & 59 & 38 \\
\hline 2 & 44 & 35 & 33 & 25 \\
\hline 3 & 37 & 19 & 29 & 8 \\
\hline 4 & 48 & 23 & 42 & 13 \\
\hline 5 & 59 & 25 & 59 & 15 \\
\hline 6 & 39 & 21 & 38 & 15 \\
\hline 7 & 53 & 24 & 47 & 15 \\
\hline 8 & 48 & 21 & 42 & 14 \\
\hline 9 & 52 & 15 & 52 & 4 \\
\hline 10 & 37 & 21 & 35 & 15 \\
\hline 11 & 44 & 33 & 34 & 27 \\
\hline 12 & 57 & 22 & 57 & 17 \\
\hline 13 & 66 & 18 & 54 & 15 \\
\hline 14 & 38 & 27 & 36 & 17 \\
\hline 15 & 40 & 12 & 32 & 13 \\
\hline 16 & 42 & 29 & 32 & 21 \\
\hline 17 & 35 & 30 & 25 & 19 \\
\hline 18 & 84 & 28 & 74 & 17 \\
\hline 19 & 37 & 29 & 36 & 30 \\
\hline 20 & 36 & 25 & 34 & 15 \\
\hline 21 & 32 & 25 & 32 & 15 \\
\hline 22 & 50 & 23 & 50 & 17 \\
\hline 23 & 37 & 36 & 32 & 26 \\
\hline 24 & 42 & 31 & 31 & 21 \\
\hline 25 & 59 & 30 & 48 & 11 \\
\hline
\end{tabular}

speed was mostly slightly higher than average car speed on the same types of roads (Department for Transport, 2006). Simulation analysis results showed that $24 \%$ motorcycles had impact speed of $50 \mathrm{~km} / \mathrm{h}$ or more and $72 \%$ motorcycles had impact speed between 25 to $50 \mathrm{~km} / \mathrm{h}$ at moment of collision. Results also showed that $80 \%$ of cars had an impact speed of $25 \mathrm{~km} / \mathrm{h}$ or less at the time of the collision.

Collision avoidance maneuver is an action performed by the participant to avoid aware/unexpected collision such as braking, swerving etc. Simulation analysis results evaluated that in car-motorcycle collisions, $84 \%$ of the car drivers did not attempt any collision avoidance maneuvers and $16 \%$ of the car drivers attempted some sort of collision avoidance maneuvers ( $0 \%$ by braking, $16 \%$ by swerving) as shown in Table 2. Results also evaluated that $56 \%$ of riders did not attempt any collision avoidance maneuvers and $48 \%$ of the riders attempted some sort of collision avoidance maneuvers ( $20 \%$ by braking, $24 \%$ by swerving). 


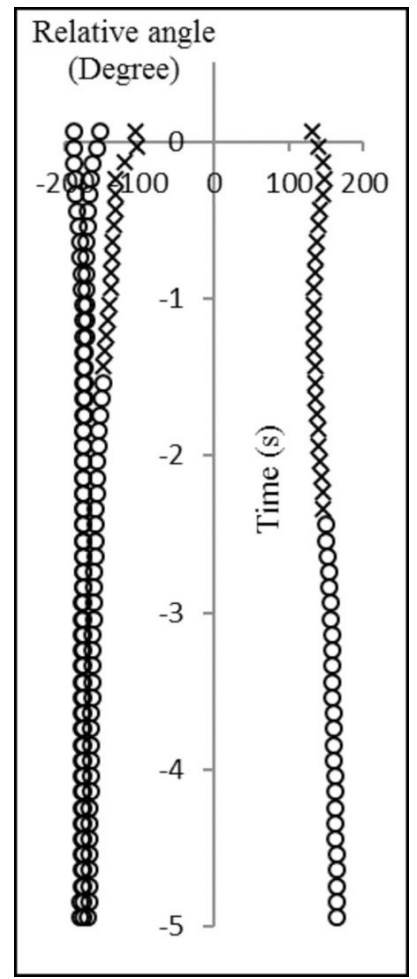

(a)

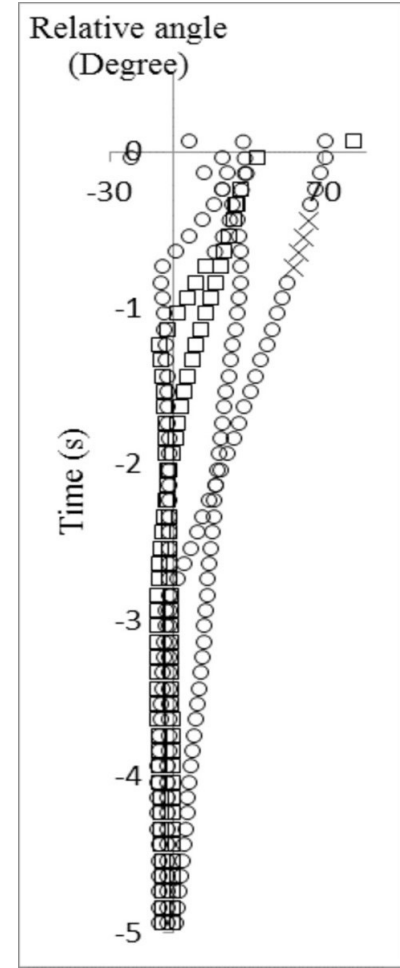

(c)

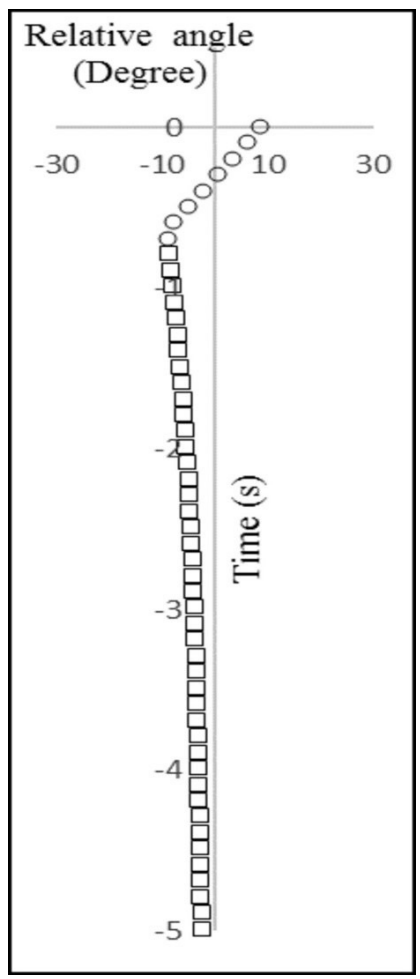

(b)

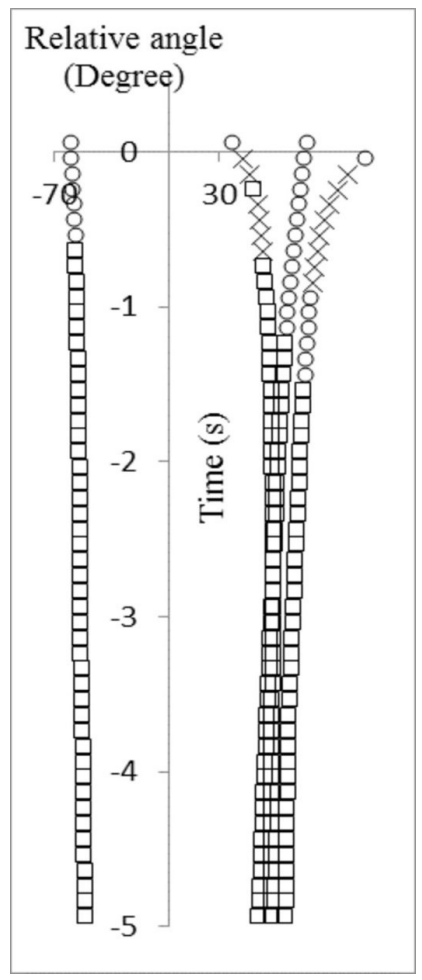

(d)

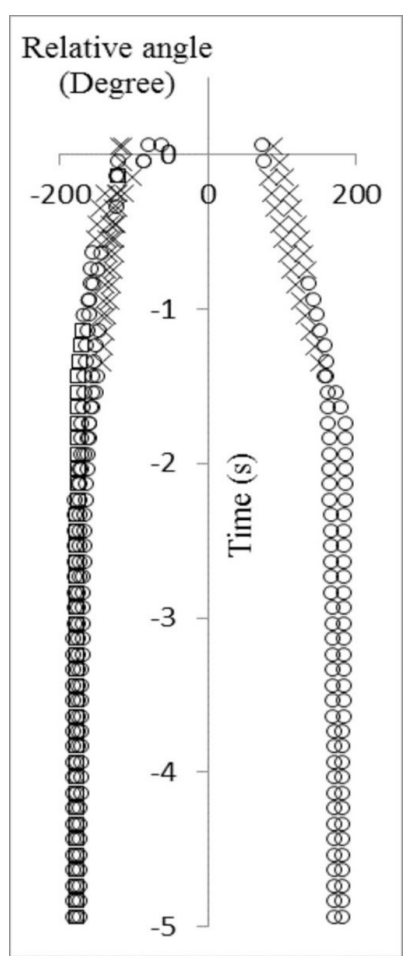

(c)

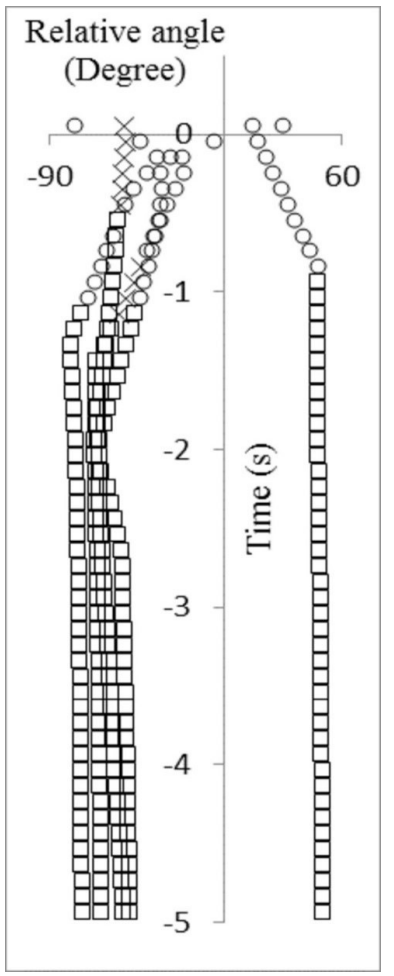

(e)

Fig. 4 (a) Same direction and straight-ahead; (b) Opposite direction and straight ahead; (c) Same direction and turning; (d) Opposite direction and turning; (e) Adjacent direction and without turning; (f) Adjacent direction and turning 
Table 2 Collision avoidance maneuvers frequency

\begin{tabular}{lcc}
\hline $\begin{array}{l}\text { Collision avoidance } \\
\text { maneuver }\end{array}$ & $\begin{array}{c}\text { Car drivers } \\
\text { (Frequency) }\end{array}$ & $\begin{array}{c}\text { Motorcycle Riders } \\
\text { (Frequency) }\end{array}$ \\
\hline $\begin{array}{l}\text { No collision avoidance } \\
\text { attempted }\end{array}$ & 21 & 14 \\
Braking & 0 & 5 \\
Swerve & 4 & 6 \\
Total & 25 & 25 \\
\hline
\end{tabular}

A better understanding of the correlation between motorcyclist injury severity and other involved factors such as crash characteristics, vehicle, human and weather, may help for the identification of appropriate countermeasures (Pai, 2009). In order to improve the detection of powered two-wheelers (PTW) by drivers, in-vehicle sensors or vehicle-to-vehicle communication could be employed to increase awareness to the drivers and provide important information (Fuerstenberg, 2005; Matheus et al., 2005).

Safety systems were proposed by considering the observed contributing factors to rider visibility issues for both car drivers and motorcycle riders' perspectives. From a car driver's perspective, timely detection of approaching motorcycle could signify a way to avoid accidents. The car-motorcycle accidents in which blind spots was observed as a contributing factor to visibility issue the proposed safety systems included in-vehicle sensors which can warn drivers before hazardous situation. The car-motorcycle accidents in which the view obstruction was observed as contributing factor to visibility issue the proposed safety systems included active safety devices (e.g. ABS, collision avoidance devices) which can provide the warning about the imminent collision or minimize the severity of the collision. For accidents in which high speed was observed as contributing factor to rider visibility issues the proposed safety systems included the vehicle to vehicle communication (V2V) which can share information between vehicles about speed, braking, the direction of travel and location. Driver training can also help car drivers to make the right decision in a hazardous situation

\section{References}

ACEM (2004) "In-depth investigations of accidents involving powered two wheelers", Brussels, Belgium, Rep. 2.

Brenac, T., Clabaux, N., Perrin, C., Van Elslande, P. (2006) "Motorcyclist conspicuity related accidents in urban areas: a speed problem?", Advances in Transportation Studies, (8), pp. 23-29. [online] Available at: http://www.atsinternationaljournal.com/index. php/2006-issues/viii-april-2006/367-motorcyclist-conspicuity-related-accidents-in-urban-areas-a-speed-problem [Accessed: 16 November 2018] to avoid the collision or minimize the severity of the collision. From a motorcycle rider perspective, the safety measures included the use of speed warning systems, high visible helmet, daytime running lights, and modified rider clothing to improve the rider visibility. The study revealed that $10 \%$ speed reduction before the collision can reduce fatal injury of car crashes by $30 \%$ (Krafft et al., 2009).

\section{Conclusion}

This paper investigated the contributing factors to rider visibility issues in car-motorcycle accidents with the help of a simulation tool and safety measures were proposed accordingly. The investigation involved a full reconstruction of the accidents on Virtual Crash software 2.2. Car-motorcycle accidents were categorized into dominant accident types based on statistical data analysis. The accidents which involved one car and one motorcycle in the built-up area were identified by accident types. Twenty-five accidents were selected by accident types for simulation analysis. The collision configuration and the orientation of the car and motorcycle were described by measuring, the relative heading angles between the car and the motorcycle at the time of the collision. The simulation analysis evaluated the main contributing factors to rider visibility issues such as view obstructions, blind spots and high speed. View obstructions (stationary and mobile) were observed higher as compared to other contributing factors to rider visibility issues. Car drivers were observed negligent about blind spots as contributing factor to visibility issues. It was also observed that the high speed of rider did increase the possibility of the rider not being seen and some drivers took the wrong decision before the collision. Simulation data plots showed the involvement and variation of contributing factors which reduce rider visibility for 5 seconds before the collision. The study found that most of the car drivers could not perform collision avoidance maneuvers such as braking and swerving due to observed rider visibility issues. Safety systems were proposed from car and motorcycle perspectives based on simulation results to solve rider visibility issues.

Brown, I. D. (2002) "A review of the 'Looked but failed to see' accident causation factor", In: Behavioural Research in Road Safety: Eleventh Seminar, London, UK, pp. 116-124.

Clarke, D. D., Ward, P., Bartle, C., Truman, W. (2004) "In-depth study of motorcycle accidents", Department for Transport, London, UK, Rep. 54.

Clarke, D. D., Ward, P., Bartle, C., Truman, W. (2007) "The role of motorcyclist and other driver behaviour in two types of serious accident in the UK", Accident Analysis \& Prevention, 39(5), pp. 974-981. https://doi.org/10.1016/j.aap.2007.01.002 
Collins Publishers "Definition of 'blind spot'", [online] Available at: https://www.collinsdictionary.com/dictionary/english/blind-spot [Accessed: 23 November 2018]

Department for Transport (2006) "Transport Statistics Bulletin Compendium of Motorcycling Statistics", 32nd ed., The Stationery Office, London, UK.

Fuerstenberg, K. Ch. (2005) "A new European approach for intersection safety - the EC-project INTERSAFE", In: 8th International IEEE Conference on Intelligent Transportation Systems, Vienna, Austria, pp. 432-436. https://doi.org/10.1109/ITSC.2005.1520072

Green, M. (2000) "'How Long Does It Take to Stop?" Methodological Analysis of Driver Perception-Brake Times", Transportation Human Factors, 2(3), pp. 195-216. https://doi.org/10.1207/STHF0203_1

Herslund, M.-B., Jorgensen, N. O. (2003) "Looked-but-failed-tosee-errors in traffic", Accident Analysis \& Prevention, 35(6), pp. 885-891. https://doi.org/10.1016/S0001-4575(02)00095-7

Jacobs, G., Aeron-Thomas, A., Astrop, A. (2000) "Estimating Global Road Fatalities", Transport Research Laboratory, Crowthorne, Berkshire, UK, Rep. 445.

Kim, K., Boski, J. (2001) "Finding fault in motorcycle crashes in Hawaii- environmental, temporal, spatial, and human factors", Transportation Research Record, 1779(1), pp. 182-188. https://doi.org/10.3141/1779-24

Krafft, M., Kullgren, A., Lie, A., Strandroth, J., Tingvall, C. G. (2009) "The effects of automatic emergency braking on fatal and serious injuries", In: International Conference on the Enhanced Safety Vehicles (ESV), Stuttgart, Gemany, Paper No. 09-O419.

Matheus, K., Morich, R., Paulus, I., Menig, C., Lübke, A., Rech, B., Specks, W. (2005) "Carto-car communication-market introduction and success factors", presented at ITS'05: 5th European Congress and Exhibition on Intelligent Transport Systems and Services, Hannover, Germany, 2005.
Molinero Martinez, A., Carter, E., Naing, C. L., Simon, M. C., Hermitte,T. (2007) "Accident causation and pre-accidental driving situations. Part 1. Overview and general statistics", Traffic Accident Causation in Europe [TRACE], Deliverable D 2.1, Project No. 027763.

Nielsen, S. K. (2009) "Motorcycle accidents", AIB, Danish Road Traffic Accident Investigation Board, Rep. 6.

Pai, C.-W. (2009) "Motorcyclist injury severity in angle crashes at T-junctions: Identifying significant factors and analyzing what made motorists fail to yield to motorcycles", Safety Science, 47(8), pp. 1097-1106. https://doi.org/10.1016/j.ssci.2008.12.007

Pierini, M., Cappon, H., König, J., Garcia, B., Lopez-Valdes, F. (2004) "Motorcyclists: Accident national data", AP-SP41-0001-C, Report, APROSYS SP4 - TIP3-CT-2004-50650.

Seyer, K., Newland, C., Terrell, M., Dalmotas, D. (2000) "The effect of mass, stiffness and geometry on injury outcome in side impacts: a parametric study", In: Proceeding of the 44th Stapp Car Crash Conference, Atlanta, Georgia, pp. 6-8. https://doi.org/10.4271/2000-01-SC01

Tang, K.-H. (2003) "A field study on validation of supplemental brake lamp with flashing turn signals for motorcycles", International Journal of Industrial Ergonomics, 31(5), pp. 295-302. https://doi.org/10.1016/S0169-8141(02)00219-6

Wach, W. (2016) "Calculation reliability in vehicle accident reconstruction", Forensic Science International, 263, pp. 27-38. https://doi.org/10.1016/j.forsciint.2016.03.038

Watanabe, R., Katsuhara, T., Miyazaki, H., Kitagawa, Y., Yasuki, T. (2012) "Research of the relationship of pedestrian injury to collision speed, car-type, impact location and pedestrian sizes using Human FE Model", In: Proceeding of the 56th Stapp Car Crash Conference, Savannah, Georgia, pp. 29-31. https://doi.org/10.4271/2012-22-0007 